Mediterránea Ser. Biol. (1990), n. 12. Pág. 71-78

\title{
COLEÓPTEROS CARÁBIDOS ASOCIADOS A LOS CAMPOS DE CULTIVO DE LA CAMPIÑA CORDOBESA $\left({ }^{*}\right)$
}

\author{
por \\ ANA M. ${ }^{a}$ CÁRDENAS TALAVERÓN( ${ }^{(1)}$ y CARMEN BACH PIELLA ${ }^{(1)}$
}

\section{RESUMEN}

Este trabajo constituye parte de un estudio realizado sobre la población de artrópodos asociada a los campos de cultivo de la campiña cordobesa.

Las investigaciones se están efectuando en unos terrenos situados a 7 kilómetros de Córdoba, en dirección SW, dedicados al cultivo alternativo de trigo y de girasol.

La fauna epigea fue recolectada mediante trampas de caída libre y Berlese.

Un total de 23 especies de carábidos fueron capturadas de marzo a agosto, período de duración de los muestreos.

El análisis de los resultados pone de manifiesto una escasez de especies fitófagas frente a las depredadoras y una preferencia por suelos arcillosos frente a los arenosos.

\section{SUMMARY}

This is part of a study on the epigeal arthropod fauna found in cultivated fields in Cordoba (SW Spain).

The research is being carried out in a field located $7 \mathrm{Km}$. (SW) from Cordoba and cultivated with alternative weat and sundflower crops.

The epigeal fauna was collected either by pitfall traps or Berlese.

A total of 23 species of carabid beetles were cought between March and August 1986 (sampling period).

The results showed less phytophagous species when compared the predatory ones as well as a preference to clay soil when compared the sandy ones.

PALABRAS CLAVE: Coleópteros, Carábidos, Córdoba.

KEY WORDS: Coleopteran, Carabid Beetles, Cordoba.

(1) Departamento de Biología Animal (Zoologia). Facultad de Ciencias. Universidad de Córdoba. 14071. Córdoba.

$\left(^{*}\right.$ Trabajo incluido en el Proyecto PB-86-0165.

$(*)$ Este trabajo ha sido subvencionado por la C.A.I.C.T.Y.T. en el contexto del Proyecto de Investigación n. ${ }^{\circ}$ PR84-0921-C02-02. 


\section{INTRODUCCIÓN}

Este trabajo forma parte de un proyecto de investigación que se está llevando a cabo acerca de la composición y estructura de la entomofauna asociada a los campos de cultivos extensivos de la campiña cordobesa, con el fin de poder determinar la influencia de la comunidad de insectos sobre la productividad de dichos campos.

Dentro de la fauna de insectos en general y de la de coleópteros en particular, la familia Carabidae desempeña un papel fundamental debido, por un lado, a la posible incidencia de las especies consideradas nocivas sobre la productividad de dichos campos y, por otro, al posible efecto de control biológico que otras especies de dicha familia, por su condición depredadora, puedan realizar.

La zona de estudio (que queda incluida en la finca denominada «El Judío Bajo») la integran unos terrenos situados a 7 kilómetros de Córdoba capital, en dirección suroeste, con unas cotas de altitud comprendidas entre los 250 y $280 \mathrm{~m}$. y unas coordenadas U.T.M. 30 S UG 510890-520900, dedicados tradicionalmente a cultivos extensivos de cereales (trigo), alternando anualmente con girasol para su explotación.

\section{METODOLOGIA}

Se han delimitado dos parcelas en el área de estudio, denominadas El Lejío (sembrada de trigo) y El Huerto (sembrada de girasol) (ver Fig. 1), en cada una de las cuales se han dispuesto 12 trampas de caída libre distribuidas en función del acceso que permitía el cultivo, a fín de no dañarlo durante los muestreos, según se indica en el esquema de la mencionada figura 1 , y cuyo diseño se muestra en la figura 2.

Los recipientes de las trampas se llenaron con 250 c.c. de una solución de formol al $1 \%$ y detergente al $10 \%$, alternando semanalmente con ácido pícrico al $0,3 \%$ y detergente al $10 \%$, a fin de comprobar la efectividad de ambos productos, ya que diversos autores recomiendan el empleo de uno u otro (PIETRAZSKO y CLERK, 1980, el formol, y LOREAU, 1983, el pícrico). Las trampas se vaciaron y rellenaron semanalmente en los campos de trigo y/o en los de girasol, según la fenología del cultivo durante el período de marzo a agosto.

Al mismo tiempo se recogieron muestras de cultivo y tierra, que se llevaron al laboratorio para la extracción de fauna edáfica siguiendo la técnica Berlese-Tullgren (MARCOS y CIA, 1988).

Para la determinación de las especies de carábidos se ha seguido el mismo criterio sistemático que NOVOA (1974), basándonos para la definición de tribus en GANGLBAUER (1892) y para la de géneros en JEANNEL $(1941,42)$.

\section{RESULTADOS Y DISCUSIÓN}

A continuación se relacionan las especies de carábidos recolectados en la zona durante el período de muestreos. 

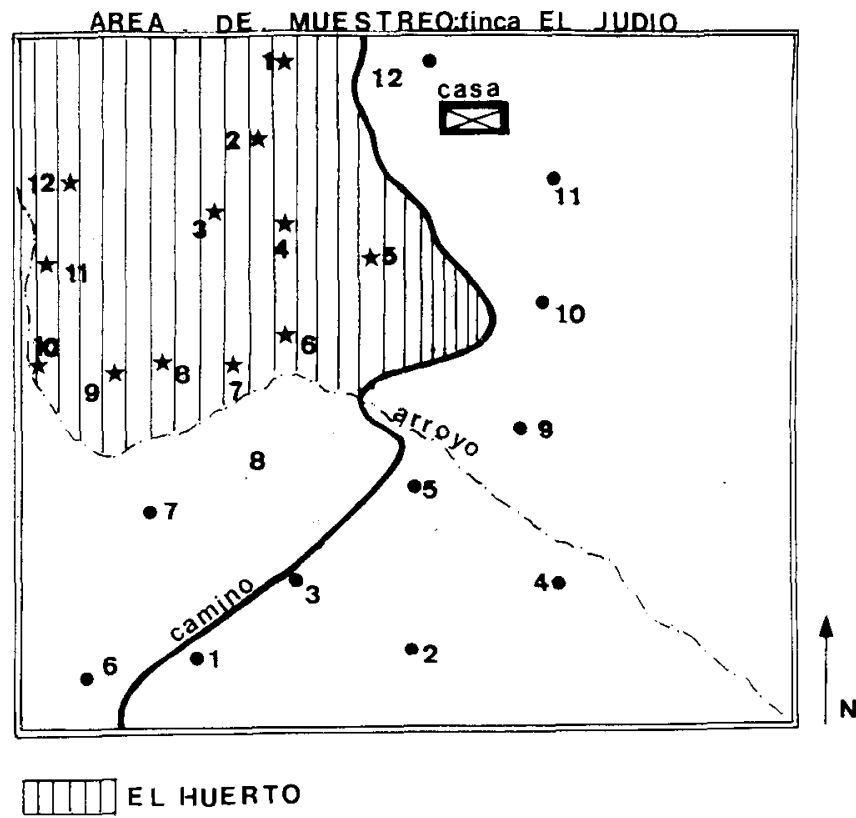

JEL LEJIO

Figura 1.-Esquema de la parcela en estudio, marcando con diferente trama el área correspondiente a cultivos de trigo y de girasol. Los asteriscos y los puntos indican la situación de las trampas en cada uno de los cultivos.

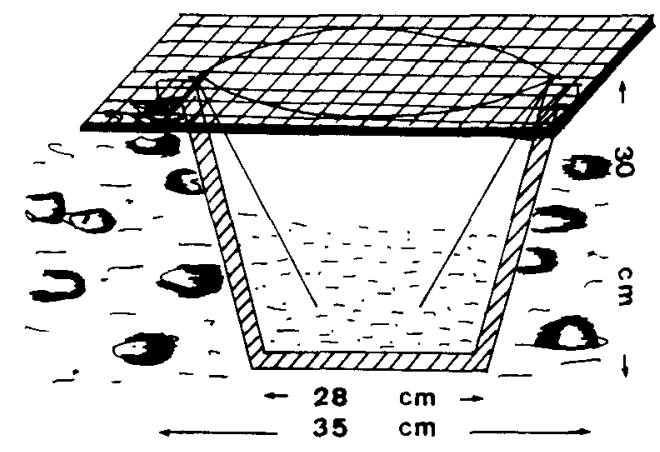

Trampa de caida

Figura 2.-Esquema de una trampa de caída. 


\section{Tribu notiophilini}

Notiophilus geminatus Dejean, 1831.

El Lejío, 24-III-86, 10 .

Notiophilus quadripunctatus Dejean, 1826.

El Lejío, 7-VI-86, 10 .

\section{Tribu bembidiini}

Tachys scutelaris dimidiatus Motschoulsky, 1849.

El Lejío, 28-IV-86, 10", 300कo; 1-V-86, 1\%; 8-IV-86, 10 .

Princidium (Testedium) laetum Brullé, 1838.

El Lejío, 28-IV-86, 20"0', 1 . .

Metallina (Neja) ambiguum (Dejean, 1831).

El Lejío, 1-V-86, 1\%; 28-V-86, 40"O', 1\%; 7-VI-86, 1 \%.

Philochtus lunulatus (Fourcroy, 1785.

El Huerto, 30-III-86, 2कo; 9-V-86, $10^{\circ}$.

El Lejío, 2-V-86, $10^{\circ}$.

\section{Tribu Pterostichini}

Anchus ruficornis Goeze, 1777.

El Huerto, 9-V-86, 1 .

Calathus (Calathus) ambiguus (Paykull, 1790).

El Huerto, 24-III-86, 1 .

Orthomus barbarus (Dejean, 1828).

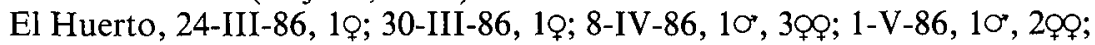
20-VI-86, 1 \%.

Poecilus (Parapoecilus) kugelanni (Panzer, 1797).

El Lejío, 1-V-86, 10", 2oి.

Poecilus (Pseudopedius) crenatus (Dejean, 1838).

- El Lejío, 8-IV-86, 1 \%.

El Huerto, 1-V-86, 10;, 1\%; 7-V-86, 10", 1 . .

Argutor (Omaseus) aterrimus nigerrimus (Dejean, 1828).

El Lejío, 30-III-86, 1 .

El Huerto, 17-IV-86, 10, 1థ; 1-V-86, 10, 1\%.

\section{Tribu harpalini}

Egadroma marginatum (Dejean, 1829).

El Huerto, 8-V-86, 10 .

Acupalpus brunneipes (Sturm, 1825).

El Huerto, 28-IV-86, 10, 6థ९; 8-V-86, 1९; 9-V-86, $10^{\circ}$.

Carterus fulvipes (Latreille, 1817).

$1-\mathrm{V}-86,10$.

Carterus (Odontocarus) cephalotes (Dejean, 1826).

El Huerto, 20-VI-86, 10", 1\%; 10-VII-86, $10^{\circ}, 1$ \%. 
Scybalicus oblongiusculus (Dejean, 1829).

El Huerto, 1-V-86, 1 \%.

\section{Tribu Lebiini}

Demetrias atricapillus (Linné, 1758).

El Lejío, 8-V-86, 10 .

Syntomus fuscomaculatus Motschoulsky, 1844.

El Lejío, 1-VI-86, 10.

Mesolestes scapularis (Dejean, 1829).

El Huerto, 8-IV-86, 10; 17-IV-86, 10; 1-V-86, 10; 16-VII-86, 10*.

El Lejío, 9-V-86, 10;; 20-VI-86, 1 . .

Microlestes ibericus (Holdhaus, 1912).

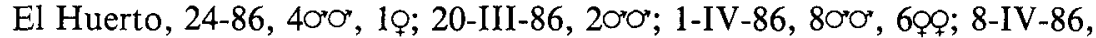

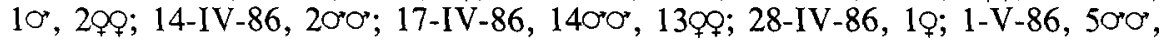

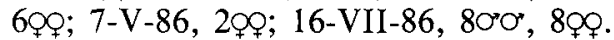

El Lejío, 10-VII-86, $10^{\circ}$.

Microlestes abeillei (Brisout, 1885).

El Huerto, 1-VI-86, 10, 1\%; 17-IV-86, 60\% VII-86, 200', 1 \%.

El Lejío, 8-IV-86, 20̛ơ; 1-VII-86, 1 \%.

Microlestes luctuosus Holdhaus, 1904.

El Huerto, 8-IV-86, 1\%; 17-IV-86, 20\%०, 1 \%.

El Lejío, 1-V-86, 2 क्干.

En total, son 23 las especies de carábidos halladas, dos de ellas (del género Notiophilus) pertenecientes a la subfamilia Carabinae y el resto a la Harpalinae. El $18 \%$ de la carabofauna ha sido recolectado en la parcela denominada El Lejío, en tanto que el $82 \%$ restante corresponde a El Huerto. En relación a este hecho, hay que tener en cuenta que el tipo de suelo afecta de modo considerable a la composición cualitativa y cuantitativa de la carabofauna de los biotopos agrarios (HEYDEMAN, 1955; KIRCHNER, 1960, SCHERNEY, 1960 y PIETRASZKO y CLERQ, op. cit.). Diversos autores piensan que la presencia de carábidos en un biotopo depende fundamentalmente del microclima (PAUER, 1975), el cual está altamente determinado por el tipo de suelo que afecta al grado de humedad, de evaporación, densidad de plantas... En este sentido, PIETRASZKO y CLERQ (1981) encontraron una preferencia por parte de los carábidos hacia suelos arcillosos frente a los limosos. Esta preferencia se manifiesta también en las especies que colonizan el área de estudio, por ser éstas más abundantes en la parcela El Huerto, de textura limoarcillosa (datos propios, a partir de los correspondientes análisis edafológicos), que en la de El Lejío, de textura limoarenosa.

En conjunto, se aprecia que la fauna de carábidos está escasamente representada, ya que las 23 especies determinadas suponen menos de un $3 \%$ del total de las especies peninsulares de carábidos, que se estima en cerca del millar de especies. No obstante, al comparar con los resultados obtenidos por CASTANNERA y DEL ESTAL (1983) en un estudio sobre los campos de trigo de invierno en el Sistema Central, hallaron un total de 30 especies durante dos 
años de prospecciones, y PIETRASZKO y DE CLERQ (1981) capturaron 45 especies en campos de cultivo de Bélgica, muestreando durante cuatro años, por lo que es presumible que en los biotopos racionalizados para la explotación agrícola, la comunidad de carábidos (en función de las características biológicas del grupo) se vea altamente alterada, permaneciendo únicamente aquellas especies que presentan mayor plasticidad ecológica.

También, y desde el punto de vista cuantitativo, los carábidos son más abundantes en los campos del centro peninsular que en la campiña cordobesa, lo cual es explicable en función de las diferencias climáticas, dado que la mayoría de los carábidos prefieren e incluso determinadas especies son exclusivas de zonas con cotas de temperatura muy moderadas y elevados índices de humedad, siendo más escasas las especies de carácter termófilo, que son, por otra parte, las capaces de colonizar nuestra zona.

Analizando la fluctuación temporal de la comunidad de carábidos del área de estudio (Fig. 3), se aprecia que el número de efectivos decrece de modo considerable en junio y comienza a ascender de nuevo hacia mediados de julio. La siega de los trigos y la falta de humedad, junto a las elevadas temperaturas, pueden ser las responsables del descenso. Posteriormente, las especies de los géneros Microlestes y Syntomus, de carácter termófilo efectúan un reemplazamiento dentro de la comunidad, y son, por lo tanto, las que originan el ascenso en los niveles de abundancia.

En base a un estudio de la distribución biogeográfica, biología y hábitos alimentarios, a partir de datos bibliográficos (JEANNEL, 1941, 1942; ANTOINE, 1955, 1962; FREUDE et al., 1976; JEANNE, 1965-1980; MATEU, 1963) y de las propias comprobaciones efectuadas en el campo, hemos llegado a las siguientes conclusiones:

La mayoría de las especies son bastantes generalistas, de amplia distribución peninsular, biogeográficamente mediterráneas o bético-rifeñas y de régimen alimentario carnívoro o depredador en todos o en algunos de sus estadios vitales, siendo sus presas colémbolos y otros pequeños artrópodos de la fauna epigea. En otros países se ha comprobado que algunos Poecilus y Harpalus (SUNDERLAND y VICKERMAN, 1975) son depredadores del cereal, existiendo la posibilidad de utilizar este componente biótico en el control integrado de la plaga de áfidos (LOUGHRIDGE y LUFF, 1983).

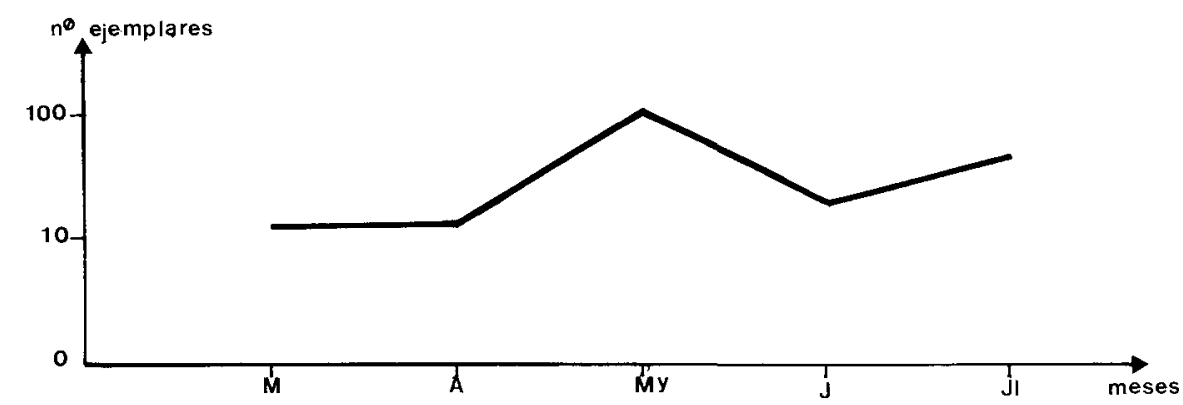

Figura 3.-Fluctuación mensual del número de ejemplares capturados. 
Existen, además, fitófagos típicos, tanto en fases larvarias como de imagos, que se alimentan casi exclusivamente de plántulas del cultivo e incluso de los granos de siembra en sus primeros ciclos, devorando las espigas de gramineas en su fase adulta. Es el caso de Carterus fulvipes y C. cephalotes. La densidad de estas especies frente al colectivo de insectos es muy baja, lo que indica que no afecta, o lo hace muy escasamente a la productividad del campo.

Otras de las especies capturadas en la zona son ripícolas o silvícolas y de presencia accidental, hallándose en microhabitats concretos de características húmedas y umbrías.

En resumen, hay dos hechos destacables en cuanto a la fauna de carábidos se refiere:

1.-Escasez de especies fitófagas (granívoras) y dañinas para los cereales. Esta escasez está determinada por dos condicionantes (CÁRDENAS y BACH, en prensa): existencia de una fauna epiedáfica de depredadores suficientemente elevada (arañas, estafilínidos...) y una competencia intra o interespecífica de especies fitófagas (elatéridos y sífidos).

2.-Presencia de carábidos depredadores que pueden actuar controlando otras poblaciones de parásitos del cereal (áfidos).

Con respecto a la efectividad del líquido conservante empleado en las trampas (ver metodología) y dado que se utilizaban alternativamente el ácido pícrico y el formol cada semana, al proceder a la separación y estudio de las muestras se apreciaban diferencias mínimas, ya sean de tipo cualitativo o cuantitativo, con la aplicación de uno u otro producto.

Estimamos que las diferencias responden más a la propia aleatoriedad del ensayo, que a un efecto selectivo de atracción o repulsión de las sustancias conservantes, por lo que no hemos considerado necesaria la aplicación de tests para determinar los niveles de significación. 


\section{BIBLIOGRAFÍA}

ANTOINE, M., 1955: Coléoptères Carabiques du Maroc (1 ${ }^{\text {re }}$ partie). Mem. Soc. Scien. Nat. Phys. Maroc (N. S.) Zool.; 1: 1-176.

- 1962: Coléoptères Carabiques du Maroc (5ème partie). Mem. Soc. Scien. Nat. Phys. Maroc (N. S.) Zool.; 9: 534-694.

CÁRDENAS, A. M., y BACH, C., en prensa: Composición de una Biocenosis cerealista de la campiña cordobesa. Datos preliminares. Act. VII Reunión Bienal de la R.S.E.H.N. Pamplona.

CASTAÑERA, P., y DEL ESTAL, P. 1983: Carábidos (Col. Carabidae) de un campo de trigo de invierno de la zona centro. Act. I Con. Ib. Entomol.: 141-174.

FREUDE, H.; HARDE, K. W., Y LOTSE, G. A. 1976: Die Käfer Mitteleuropas. Band 2. Adephaga I. Goeke \& Evers Krefeld: 301 págs.

GANGLBAUER, M. 1892: Die Käfer von Mitteleuropas. 1, Familierinche Caraboidea. Carl Gerold's wien. 557 págs.

HEY DEMANN, B. 1955: Carabiden der kulturfeder als ökologische indicatoren. Ber 7. Wanderversamm. Deut. Entomol.: 172-185.

JEANNE, C. 1965: Carabiques de la Peninsule Ibérique (2 ème note). Act. Soc. Lin. Bordeaux, 102, Ser. A, 10.

- 1980: Carabiques de la Peninsule Ibérique (2 ${ }^{\text {ème }}$ note). Bull. Soc. Lin. Bordeaux. 8: 21-47.

JEANNEL, R. 1941: Faune de France. Col. Carabiques. 39 Lib. Fac. Sci. 571 págs.

- 1942: Faune de France. Col. Carabiques. 40. Lib. Fac. Sci. 603 págs.

KIRCHNER, H. 1960: Untersuchungen zür ökologie feldbewohnender Carabiden Dissertation. Cologne.

LOREAU, M. 1983: Trophic role of Carabid Beetles in a forest. New Trends in Soil Biology. VIII Intl. Coll. of Sio. Zool. 281-286.

MARCOS GARCÍA, M. A. 1988: Métodos generales de captura. En bases para un curso práctico de Entomología. Ed. Asoc. Esp. Ent. Salamanca: 12-24.

MATEU, J. 1963: Monografía de los Microlestes (Col. Lebiinae). Mus. Roy. de l'Africa Centrale. Terburen. Belgica. Ser. 8. Sci. Zool. 121: 149 págs.

NOVOA, F. 1974: Los Carabidae de la S. ${ }^{a}$ de Guadarrama. Tesis Doctoral. Univ. Comp. de Madrid.

PAUER, R. 1975. Zur Ausbreiton der Carabiden in der Agrarlendschaft. Z. Angew. Zoll. 62: 457-489.

PIETRASZKO, R., y DE CLERQ, R. D. 1980: Ėtudes de la population d'artropodes epigés dans les cultures au cours de la periode 1974-1978. Rev. L'Agriculture, 4 (3): 720-733.

- 1981: Carabidae of arable land in Belgium. Stat. Nema. en Parasitica, 37 (2): 45-58.

SCHERNEY, A. M. 1960: Beitrage zur biologie und okonomischen Bedeutung rauberischlebender kaferarten in Feldkulturen (Teil II) Z. Angew. Entomol. 47: 231-255.

SUNDERLAND, K. D., y VICKERMAN, G. P. 1975: Arthropods in cereal crops nocturnal activity, vertical distribution and aphid predation. J. Appl. Ecol., 12: 755-765. 